PROCEEDINGS OF THE

AMERICAN MATHEMATICAL SOCIETY

Volume 130, Number 1, Pages 157-161

S 0002-9939(01)06030-0

Article electronically published on May 3, 2001

\title{
A GLOBAL PINCHING THEOREM FOR SURFACES WITH CONSTANT MEAN CURVATURE IN $S^{3}$
}

\author{
YI-JUNG HSU AND TAI-HO WANG \\ (Communicated by Christopher Croke)
}

\begin{abstract}
Let $M$ be a compact immersed surface in the unit sphere $S^{3}$ with constant mean curvature $H$. Denote by $\phi$ the linear map from $T_{p}(M)$ into $T_{p}(M), \phi=A-\frac{H}{2} I$, where $A$ is the linear map associated to the second fundamental form and $I$ is the identity map. Let $\Phi$ denote the square of the length of $\phi$. We prove that if $\|\Phi\|_{L^{2}} \leq C$, then $M$ is either totally umbilical or an $H(r)$-torus, where $C$ is a constant depending only on the mean curvature $H$.
\end{abstract}

\section{INTRODUCTION}

Let $M$ be a compact immersed hypersurface in the unit sphere $S^{n+1}$ with constant mean curvature $H$. Denote by $h=\left[h_{i j}\right]$ the second fundamental form of $M$ and by $\phi$ the tensor $\phi_{i j}=h_{i j}-\frac{H}{n} \delta_{i j}$. Let $\Phi$ denote the square of the length of $\phi$. It is well known that if $H=0$ and $0 \leq \Phi \leq n$, then $M$ is either the equatorial sphere or a Clifford torus [3]. Recently, H. Alencar and M. do Carmo extended the above result to a hypersurface $M$ with constant mean curvature $H$ [1]. They proved that $M$ is either totally umbilical or an $H(r)$-torus if $\Phi$ satisfies a certain pointwise pinching condition. In 1989, C. L. Shen proved that a minimal hypersurface $M$ is totally geodesic if $M$ is of nonnegative sectional curvature, and $\Phi$ satisfies a certain global pinching condition [8]. Later, the first author improved a result of Shen in the case of $n=2$ and found a sharp bound concerning the global pinching condition 6]. The purpose of this paper is to extend our global theorem to a surface $M$ with constant mean curvature $H$ and obtain the best constant.

Before stating our main result, let $B$ be the constant $B=2+\frac{H^{2}}{2}$ and $m(B)$ be the maximum value of the function $q(x)=2 \sqrt{2} \frac{\sqrt{B} x^{2}+2(B-2) x+B \sqrt{B}}{(\sqrt{B}+x)^{2}\left(\left(x^{2}-B\right)^{2}+8 x^{2}\right)}$ on $[0, \infty)$. The following is our main result.

Theorem 1.1. Let $M$ be a compact immersed surface in the unit sphere $S^{3}$ with constant mean curvature $H$. Then

$$
\|\Phi\|_{2} \geq 2 \pi \sqrt{\frac{2 g}{M(B)}},
$$

Received by the editors April 17, 1997 and, in revised form, May 10, 2000.

2000 Mathematics Subject Classification. Primary 53C40, 53C42.

Key words and phrases. Mean curvature, sphere, totally umbilical.

(C)2001 American Mathematical Society 
where $g$ is the genus of $M$ and $\|\cdot\|_{2}$ is the $L^{2}$-norm. The equality holds if and only if $M$ is either totally umbilical or an $H(r)$-torus. In particular, if $\|\Phi\|_{2} \leq 2 \pi \sqrt{\frac{2}{M(B)}}$, then $M$ is either totally umbilical or an $H(r)$-torus.

It should be noted that for $M$ to be either totally umbilical or an $H(r)$-torus, $\|\Phi\|_{2}=2 \pi \sqrt{2 g}\left(\frac{B}{2}\right)^{\frac{3}{4}}$. It turns out that there exists a constant $H_{0} \geq 2$ such that $m(B)=\left(\frac{2}{B}\right)^{\frac{3}{2}}$ for all $|H| \leq H_{0}$. As a immediate consequence of the above result, we state

Corollary 1.2. Let $M$ be a compact immersed surface in the unit sphere $S^{3}$ with constant mean curvature $H,|H| \leq H_{0}$. Then

$$
\|\Phi\|_{2} \geq 2 \pi \sqrt{2 g}\left(\frac{B}{2}\right)^{\frac{3}{4}}
$$

where $g$ is the genus of $M$ and $\|\cdot\|_{2}$ is the $L^{2}$-norm. The equality holds if and only if $M$ is either totally umbilical or an $H(r)$-torus. In particular, if $\|\Phi\|_{2} \leq 2 \pi \sqrt{2}\left(\frac{B}{2}\right)^{\frac{3}{4}}$, then $M$ is either totally umbilic or an $H(r)$-torus.

For the proof of the main theorem, we shall need the following Bernstein-Hopf theorem (see [2], [5]).

Theorem 1.3. Let $M$ be a compact immersed surface in the unit sphere $S^{3}$ with constant mean curvature $H$. If $M$ is a topological sphere, then $M$ is totally umbilical.

\section{Notations AND AUXiliary RESUlts}

Let $M$ be a compact connected immersed surface in the unit sphere $S^{3}$. Following the notations of [1] and [3],

Lemma 2.1. $\frac{1}{2} \Delta \Phi=\Phi(B-\Phi)+\sum \phi_{i j k}^{2}$ where $\phi_{i j k}$ denote the covariant derivative of $\phi_{i j}$.

Lemma 2.2. $|\nabla \Phi|^{2}=2 \Phi \sum \phi_{i j k}^{2}$.

Lemma 2.3. If $\Phi \geq B$, then $\Phi$ is a constant function, $\Phi \equiv B$, and $M$ is an $H(r)$-torus.

Proof. By Lemmas 2.1 and 2.2, we have $\frac{1}{2} \Delta \log \Phi=B-\Phi$ at the points where $\Phi$ is positive. It follows that $\Phi=B$ on $M$.

According to Lemma 2.1 and Lemma 2.3, we see that if $\Phi$ is a constant function, then either $\Phi=0$ or $\Phi=B$.

In the minimal case, H. B. Lawson proved that the set of all zeros of $\Phi$ is either the whole space $M$ or at most a finite set of points [7]. We need the following analogous result for the case that $M$ is with constant mean curvature.

Lemma 2.4. The set of all zeros of $\Phi$ is either the whole $M$ or at most a finite set of points.

Proof. The proof of the lemma is similar to that of Lawson. Let us sketch the proof for completeness. We use an isothermal coordinate $(u, v)$ on a neighborhood $D$ in $M$. Denote the position vector of this immersion by $X$ and the unit normal of $M$ in $S^{3}$ by $N$. Then the mean curvature $H$ and the Gaussian curvature $K$ are given 
by $\frac{1}{F^{2}}\left(X_{u u}+X_{v v}\right) \cdot N$ and $\frac{1}{F^{4}}\left[\left(X_{u u} \cdot N\right)\left(X_{v v} \cdot N\right)-\left(X_{u v} \cdot N\right)^{2}\right]$ respectively, where $F^{2}=X_{u} \cdot X_{u}=X_{v} \cdot X_{v}$.

Since $M$ is of constant mean curvature, the Weingarten equations imply that $X_{u v} \cdot N$ is harmonic in the $(u, v)$ coordinate, $X_{u u} \cdot N$ and $X_{v v} \cdot N$ differ by a constant if $X_{u v} \cdot N$ is constant on $D$, and the zero set of $X_{u v} \cdot N$ and that of $\left(X_{u u}-X_{v v}\right) \cdot N$ intersect transversely at the points where the gradient of $X_{u v} \cdot N$ does not vanish. Let $G$ be the set of all points where the gradient of $X_{u v} \cdot N$ vanishes. Since $X_{u v} \cdot N$ is harmonic, $G$ is either isolated or the whole $D$. For $G$ being isolated, the set of all zeros of $\Phi$ is isolated. In the other case, the set of all zeros of $\Phi$ is either empty or the whole $D$.

Lemma 2.5. If $M$ is not totally umbilical, then

$$
\lim _{\epsilon \rightarrow 0} \sum_{i=1}^{k} \int_{\partial B_{\epsilon}\left(p_{i}\right)} \frac{\Phi_{r}}{\Phi}=16 \pi(g-1)
$$

where $p_{1}, p_{2}, \cdots, p_{k}$ constitute all the zeros of $\Phi$ and $\Phi_{r}$ denotes the derivative of $\Phi$ on $\partial B_{\epsilon}\left(p_{i}\right)$ in the radial direction from $p_{i}$. In particular, if $\Phi$ is positive on $M$, then $M$ is a topological torus.

Proof. At the points where $\Phi$ is positive, by Lemma 2.2, we get

$$
\Delta \log \Phi=B-\Phi
$$

Integrating (2.1) over $M_{\epsilon}=M \backslash \bigcup_{i=1}^{k} B_{\epsilon}\left(p_{i}\right)$, we get, from the Gauss equation

$$
2 K=B-\Phi
$$

where $K$ is the Gaussian curvature of $M$, the assertion by Stokes's theorem and the theorem of Gauss-Bonnet.

\section{Lemma 2.6.}

$$
\int_{M}\left(\frac{\pi}{2}+\tan ^{-1} \frac{H-\sqrt{2 \Phi}}{2}-\tan ^{-1} \frac{H+\sqrt{2 \Phi}}{2}\right)(B-\Phi)+2 \sqrt{2 \Phi} \geq 4 \pi^{2}(1+g) .
$$

Proof. Regard $M$ as an immersed surface of $\mathbb{R}^{4}$. Then the total absolute curvature of $M$ in the sense of [4] is given by

$$
\begin{aligned}
T(M)= & \int_{M} \int_{0}^{2 \pi}\left|\left(\sin \theta+\frac{H+\sqrt{2 S-H^{2}}}{2} \cos \theta\right)\left(\sin \theta+\frac{H-\sqrt{2 S-H^{2}}}{2} \cos \theta\right)\right| d \theta d V \\
& =\int_{M}\left(\frac{\pi}{2}+\tan ^{-1} \frac{H-\sqrt{2 \Phi}}{2}-\tan ^{-1} \frac{H+\sqrt{2 \Phi}}{2}\right)(B-\Phi)+2 \sqrt{2 \Phi} .
\end{aligned}
$$

By the well-known inequality of Chern-Lashof [4], we have

$$
T(M) \geq \frac{\pi^{2}}{2}\left(b_{0}+b_{1}+b_{2}\right),
$$

where $b_{i}$ is the $i$ th Betti number relative to the real field, for $i=0,1,2$. Since $M$ is two-dimensional, $b_{0}=1, b_{1}=2 g$ and $b_{2}=1$. 


\section{Proof OF MAIN RESUlts}

We are now in position to prove the main result of Theorem 1.1. We may assume that $\Phi$ is positive except possibly at a finite set of points (see Lemma 2.4). By Lemmas 2.2 and 2.6, we get

$$
\begin{aligned}
& \int_{M} 2 \sqrt{2 B}+m(B) \sum \phi_{i j k}^{2}-\left(\frac{\pi}{2}+\tan ^{-1} \frac{H-\sqrt{2 \Phi}}{2}-\tan ^{-1} \frac{H+\sqrt{2 \Phi}}{2}\right)(B-\Phi)-2 \sqrt{2 \Phi} \\
& =\int_{M} m(B) \sum \phi_{i j k}^{2}+\left[\frac{2 \sqrt{2}}{\sqrt{B}+\sqrt{\Phi}}-\left(\frac{\pi}{2}+\tan ^{-1} \frac{H-\sqrt{2 \Phi}}{2}-\tan ^{-1} \frac{H+\sqrt{2 \Phi}}{2}\right)\right](B-\Phi) \\
& =\lim _{\epsilon \rightarrow 0} \int_{M_{\epsilon}} m(B) \frac{|\nabla \Phi|^{2}}{2 \Phi}+\frac{1}{2}\left[\frac{2 \sqrt{2}}{\sqrt{B}+\sqrt{\Phi}}-\left(\frac{\pi}{2}+\tan ^{-1} \frac{H-\sqrt{2 \Phi}}{2}-\tan ^{-1} \frac{H+\sqrt{2 \Phi}}{2}\right)\right] \Delta \log \Phi \\
& =\lim _{\epsilon \rightarrow 0} \int_{M_{\epsilon}} m(B) \frac{|\nabla \Phi|^{2}}{2 \Phi}+\frac{1}{2} \nabla\left[\frac{2 \sqrt{2}}{\sqrt{B}+\sqrt{\Phi}}-\left(\frac{\pi}{2}+\tan ^{-1} \frac{H-\sqrt{2 \Phi}}{2}-\tan ^{-1} \frac{H+\sqrt{2 \Phi}}{2}\right)\right] \nabla \log \Phi \\
& -\lim _{\epsilon \rightarrow 0} \int_{\partial M_{\epsilon}}\left[\frac{2 \sqrt{2}}{\sqrt{B}+\sqrt{\Phi}}-\left(\frac{\pi}{2}+\tan ^{-1} \frac{H-\sqrt{2 \Phi}}{2}-\tan ^{-1} \frac{H+\sqrt{2 \Phi}}{2}\right)\right] \frac{\Phi_{r}}{2 \Phi} \\
& =8 \pi(1-g)\left(\frac{2 \sqrt{2}}{\sqrt{B}}-\frac{\pi}{2}\right)+\int_{M}\left[m(B)-2 \sqrt{2} \frac{\sqrt{B} \Phi^{2}+2(B-2) \Phi+B \sqrt{B}}{(\sqrt{B}+\Phi)^{2}\left(\left(\Phi^{2}-B\right)^{2}+8 \Phi^{2}\right)}\right] \sum \phi_{i j k}^{2} \\
& \geq 8 \pi(1-g)\left(\frac{2 \sqrt{2}}{\sqrt{B}}-\frac{\pi}{2}\right),
\end{aligned}
$$

where the equality holds if and only if $\Phi$ is constant. On the other hand, according to Lemma 2.6, we get

$$
2 \sqrt{2 B} \operatorname{Area}(M)+m(B) \int_{M} \sum \phi_{i j k}^{2} \geq 8 \pi^{2} g-2 \sqrt{\frac{2}{B}} 8 \pi(g-1) .
$$

By combining (2.2) with the inequality (3.1), it follows from Lemma 2.1 that

$$
m(B) \int_{M} \Phi^{2} \geq 8 \pi^{2} g+\left(B m(B)-2 \sqrt{\frac{2}{B}}\right) \int_{M} \Phi \geq 8 \pi^{2} g .
$$

It remains to show that the second assertion holds. Suppose now that $\|\Phi\|_{2} \leq$ $2 \pi \sqrt{\frac{2}{m(B)}}$. The first assertion implies that $g=0$ or 1 . If $g=1$, then $\|\Phi\|_{2}=$ $2 \pi \sqrt{\frac{2}{m(B)}}$ and $\Phi$ is a constant function. If $g=0$, then by Theorem $1.3, M$ is totally umbilical. This completes the proof of Theorem 1.1.

\section{REFERENCES}

[1] H. Alencar and M. do Carmo, Hypersurface with constant mean curvature in spheres, Proc. Amer. Math. Soc. 120 (1994), 1223-1229. MR 94f:53108

[2] S. S. Chern, On surface of constant mean curvature in a three-dimensional space of constant curvature, Geometric Dynamics (Rio de Janeiro, 1981), Lecture Notes in Math., 1007, Springer-Verlag, Berlin, New York, 1983, pp. 104-108. MR 86b:53058

[3] S. S. Chern, M. do Carmo and S. Kobayashi, Minimal submanifold of a sphere with second fundamental form of constant length, Functional Analysis and Related Fields, SpringerVerlag, 1970, pp. 59-75. MR 42:8424

[4] S. S. Chern and R. K. Lashof, On the total curvature of immersed manifolds. II, Michigan Math. J. 5(1985), 5-12. MR 20:4301

[5] H. Hopf, Differential geometry in the large, Lecture Notes in Math., 1000, Springer-Verlag, 1983, pp. 136-146. MR 85b:53001

[6] Y. J. Hsu, A global pinching theorem for compact minimal surfaces in $S^{3}$, Proc. Amer. Math. Soc. 113(1991), 1041-1044. MR 92c:53040 
[7] H. B. Lawson, Complete minimal surfaces in $S^{3}$, Ann. of Math. 92(1970), 335-374. MR 42:5170

[8] C. L. Shen, A global pinching theorem of minimal hypersurfaces in the sphere, Proc. Amer. Math. Soc. 105(1989), 192-198. MR 90c:53162

Department of Applied Mathematics, National Chiao Tung University, Hsinchu, TaiWAN

E-mail address: yjhsu@math.nctu.edu.tw

Department of Applied Mathematics, National Chiao Tung University, Hsinchu, TaiWAN

E-mail address: teich@math.sinica.edu.tw 